\title{
Construção e Evidências Psicométricas de uma Escala de Avaliação da Percepção Visual
}

\section{Construction and Psychometric Evidences of a Visual Perception Scale}

\author{
Suellen Marinho Andrade ${ }^{*}, a$, Maria Manuela Caldeira de Brito da Silva Dias ${ }^{b}$, \\ Eliane Araújo de Oliveira ${ }^{a}$, Francisco Locks Neto ${ }^{c}$, \\ Renata Maria Toscano Barreto Lyra Nogueira ${ }^{d} \&$ Natanael Antônio dos Santos ${ }^{a}$ \\ ${ }^{a}$ Universidade Federal da Paraíba, João Pessoa, Brasil, ${ }^{b}$ Instituto Piaget, Gaia, Portugal, \\ ${ }^{c}$ Universidade Federal do Rio Grande do Norte, Natal, Brasil \& ${ }^{d}$ Universidade de São Paulo, São Paulo, Brasil
}

\begin{abstract}
Resumo
Este estudo teve como objetivo construir e conhecer os parâmetros psicométricos de um instrumento para análise da percepção visual de adultos. Para a construção da escala participaram 295 adultos saudáveis, sem déficits cognitivos ou perceptivo-visuais. Nesta etapa foi formulada uma escala tetrafatorial constituída por 20 itens que avaliam quatro dimensões referentes à percepção visual: constância da forma, figurafundo, posição e relação espacial. Para obter evidências de validade foi utilizada uma amostra de 183 voluntários com boa saúde física e mental e acuidade visual normal ou corrigida. Os dados obtidos relatam a existência de concordância interjuízes, adequação semântica e significância no teste-reteste do instrumento. Os coeficientes de fidedignidade variaram de 0,84 a 0,93 . Os quatro fatores esperados foram encontrados, cada um contendo 5 itens, e juntos explicaram 57,52\% da variância do constructo. $\mathrm{O}$ instrumento apresentou parâmetros psicométricos adequados, o que pode justificar sua utilidade em pesquisas básicas e na prática clínica.
\end{abstract}

Palavras-chave: Percepção visual, avaliação psicológica, adultos.

\begin{abstract}
The main objective of this study was to develop and examine the psychometric parameters of an instrument used to evaluate adults' visual perception. The basic assumption was that visual perception includes constancy of form, figure-ground, position and spatial relation. A 4-factor solution was expected. Respondents were 183 healthy adults without cognitive or visual perception impairment. In the instrument it was possible to establish the existence of inter-judges agreement, semantic adequacy and test-retest significance. The reliability coefficients ranged from 0.84 to 0.93 . The four expected factors were found, each one containing 5 items. This solution accounted for $57.52 \%$ of the construct variance indicating that the instrument has good psychometric parameters, which suggests its applicability in scientific research and clinical practice.

Keywords: Visual perception, psychological evaluation, adults.
\end{abstract}

O homem, como um organismo visualmente sensitivo, tem grande parte de suas atividades sob o controle direto da visão, o que lhe possibilita uma interação dinâmica com o ambiente. Nessa interação, a busca por informações que lhe permitem o controle adequado de suas ações reflete o seu envolvimento com o meio e indica a relevância de concatenar a percepção-ação no desenvolvimento de sua identidade. Quanto mais essa relação se aprimora, comportamentos mais compatíveis com suas

\footnotetext{
" Endereço para correspondência: Centro de Ciências Humanas Letras e Artes, Departamento de Psicologia, Universidade Federal da Paraíba, Laboratório de Percepção, Neurociências e Comportamento, Campus I, Cidade Universitária, João Pessoa, PB, Brasil 58051900. Tel.: (83) 3216 7006; Fax: (83) 3216 7337. E-mail: suellenandrade@gmail.com
}

metas podem ser observados (Oliveira \& Rodrigues, 2005).

A redução da capacidade visual implica no detrimento da qualidade de vida decorrente de restrições ocupacionais, econômicas, sociais e psicológicas. Para a sociedade, representa encargo oneroso e perda de força de trabalho. Deve-se ressaltar, portanto, a necessidade de implementar programas de deteç̧ão de distúrbios perceptivo-visuais na prática clínica, uma vez que os custos dessas ações são incomparavelmente menores do que aqueles representados pelo atendimento a portadores de distúrbios oculares e cognitivos (Granzoto, Ostermann, Brum, Pereira \& Granzoto, 2003).

Luria (1987) descreve a percepção visual como um processo ativo de procura de informação. A estrutura percebida é analisada, havendo uma síntese dos seus 
componentes com o auxílio da linguagem. Segundo Forgus (1971), o desenvolvimento da percepção visual é um pré-requisito para a adequada conduta do indivíduo com o seu ambiente.

Em referência a este desenvolvimento, Frostig e Muller (1986) constataram que, em condições favoráveis, ele é caracterizado por um complexo de habilidades e funções diferentes e relativamente independentes umas das outras. Estes pesquisadores desenvolveram mecanismos para analisar mais consistentemente a percepção, considerando o desenvolvimento de cinco áreas perceptivas: percepção viso-motora, figura-fundo, posição no espaço, relações espaciais e constância da forma.

Seguindo essa proposta de áreas perceptivas, várias escalas analisam o desenvolvimento da percepção visual, principalmente em crianças. Na década de 60 , Marianne Frostig (1964) elaborou o DTVP (Developmental Test of Visual Perception), o qual foi aplicado em mais de 6 milhões de crianças de 4 a 6 anos nos Estados Unidos. Este instrumento analisa cinco categorias da percepção visual: coordenação visuo-motora, figura-fundo, constância da forma, posição e relação espacial (Deliberato, 2000).

Posteriormente, Hammill, Pearson e Voress (1993) revisaram o DTVP e construíram uma nova versão do instrumento, o DTVP-2, o qual foi aplicado em 1.972 crianças americanas de 6 a 10 anos. Além de apresentar algumas alterações do instrumento original desenvolvido por Frostig (1964), o DTVP 2 substitui o parâmetro coordenação visuo-motora pela categoria óculo-motora. Este grupo é assim nominado por estar mais de acordo com as modalidades abarcadas na tarefa do que com o tipo de percepção visual envolvida. Este instrumento apresenta adequada confiabilidade e evidências de validade testada por vários estudos (Kaiser, Albaret \& Doudin, 2009; Moryosef-Ittah \& Hinojosa, 2006).

Tanto o DTVP quanto o DTVP-2 foram desenvolvidos na língua inglesa o que, em certa medida, exige maior adequação para amostras cuja língua materna seja outra, como é o caso da população brasileira. Contudo, há um instrumento relacionado à avaliação da percepção visual que, embora tenha sido elaborado na Europa, foi construído em língua portuguesa. O Programa de Treinamento da Percepção Visual (PTVP), desenvolvido por Dias e Chaves (2000a), é um instrumento com recursos de imagem destinado a crianças com idades entre 6 e 9 anos com deficiência de aprendizagem e distúrbios da percepção visual. Este programa foi aplicado em vários estudos envolvendo escolares e apresentou resultados consistentes com a estimulação de padrões perceptivovisuais minorados e associados com déficits de aprendizagem. As crianças submetidas ao programa, quando comparadas ao grupo controle, apresentaram evolução em várias dimensões, tais como melhora na coordenação óculo-manual, capacidade de cópia e percepção de objetos com variadas formas, identificação de figura-fundo, orientação de posição e relações espaciais bem como da velocidade visual motora e da capacidade de fechamento visual (Dias, 2009; Dias \& Chaves 2000b, 2001).

Para avaliação da percepção visual de adultos, destacam-se testes como o DTVP-A (Developmental Test of Visual Perception - Adolescent and Adult), desenvolvido por Reynolds, Pearson e Voress (2002), que se baseia nos princípios do DTVP clássico, elaborado por Frostig (1964). O DTVP-A é composto por uma bateria de 6 subtestes para medir habilidades perceptivas visuais e motoras de adolescentes e adultos. Outro teste usualmente referenciado na literatura é o CTMT (Comprehensive Trail Making Test), desenvolvido em 1938 por Partington e com evidências psicométricas para população americana (Gray, 2006). Este é um instrumento utilizado para avaliação neuropsicológica, envolvendo parâmetros de mensuração relacionados à velocidade visual motora, atenção e processamento cognitivo (Gray, 2006).

No Brasil, Noronha e Primi (2005) publicaram um estudo cujo objetivo foi identificar os instrumentos psicológicos mais conhecidos e utilizados por psicólogos brasileiros. A pesquisa envolveu 304 participantes das regiões Norte, Nordeste, Centro-oeste, Sul e Sudeste e apontou que, relacionado à percepção visual, os testes mais populares no país foram: DTVP-2, Teste Cubos de Kohs, Teste de Figuras Complexas de Rey e Teste Guestáltico Visuomotor de Bender.

O DTVP-2, desenvolvido por Hammill et al. (1993), é voltado para análise da percepção visual de crianças. Já o Teste Cubos de Kohs, que teve suas propriedades psicométricas testadas em uma amostra de 30 crianças por Toni (2010), avalia a inteligência espacial e sua relação com várias habilidades visuo-espaciais.

O Teste de Figuras Complexas de Rey foi idealizado por André Rey em 1942. As evidências de validade para este teste foram obtidas por M. Oliveira, Rigoni, Andretta e Moraes (2004) em uma amostra de 515 participantes de 5 a 15 anos de idade. Este instrumento é empregado para investigar a memória visual, a habilidade visuo-espacial e algumas funções de planejamento e execução de ações. O Teste Guestáltico Visuomotor de Bender para crianças foi originalmente construído por Lauretta Bender, em 1938, com o propósito de fornecer uma avaliação psicológica segundo os princípios da teoria gestáltica (Santos \& Jorge, 2007).

Vários sistemas alternativos de correção têm sido desenvolvidos no Brasil com intuito de buscar evidências de validade e precisão do teste de Bender (Bartholomeu, Rueda, \& Sisto, 2005; Pinelli \& Pasquali, 1990). Dentre eles, encontra-se o estudo de Posada (2002) que desenvolveu um sistema de classificação das respostas fundamentado nos princípios piagetianos de construção e representação espacial. Os resultados obtidos com aplicação de seu sistema indicaram bons parâmetros psicométricos e vantagens em relação ao método de Koppitz. Também nesse sentido, Sisto, Noronha e San- 
tos (2006) desenvolveram o teste Gestáltico Visomotor de Bender - Sistema de Pontuação Gradual (B-SPG), tendo como embasamento os pressupostos teóricos do Bender. Os dados obtidos demonstraram a sensibilidade do B-SPG para a captação do caráter maturacional do desenvolvimento psicomotor, indicando que com o aumento da idade, as distorções da cópia progressivamente diminuem.

Segundo Silva e Nunes (2007), Bender analisou, ainda, a produção do Teste Guestáltico Visuomotor em pacientes adultos, com algum tipo de afasia ou apraxia, sendo constatados erros de movimento e percepção do estímulo. Entretanto, muitas pesquisas, realizadas internacionalmente e no Brasil, usam impressões clínicas gerais quando avaliam protocolos do Bender, não tendo, contudo, uma correção padronizada do teste. Conforme sugerem os autores, a elevada subjetividade na interpretação dos resultados frente ao teste colabora para reduzir a confiança no mesmo.

Contudo, contrapondo-se a estes resultados, pode ser citado o estudo de Bandeira e Hutz (1994), por exemplo, que investigaram o grau de predição do rendimento escolar por meio da aplicação dos testes Desenho da Figura Humana (DFH), Bender e Raven. Os dados obtidos apontaram para correlações significativas entre os três testes e o rendimento, embora apenas o Bender e os itens evolutivos do DFH tenham contribuído para explicar a variância do rendimento escolar. Do mesmo modo, vale destacar o estudo desenvolvido por Sisto, Bueno e Rueda (2003) que analisaram traços de personalidade em crianças sem histórico clínico, em relação à escala de maturação neurológica. Segundo os autores, a aplicação do teste de Bender permitiu constatar que os elementos estruturais gestálticos parecem guardar relações com sintomas psicopatológicos.

A maioria das pesquisas desenvolvidas no Brasil, relacionadas à percepção visual de adultos, aplica instrumentos como o VOSP (Visual Object Space Perception), o VFD (Visual Form Discrimination Test) e o JOL (Judgment of Line Orientation; Caixeta \& Nitrini, 2001; C. Oliveira, Rodrigues, \& Fonseca, 2009; Quental, Brucki, \& Bueno, 2009; Teixeira, 2008) que não apresentam normatização e análise de validade ou confiabilidade para nossa população.

Em 2005, Toselo realizou um estudo de normatização do Teste VOT (Hooper Visual Organization Test) para a população brasileira. O VOT é um instrumento utilizado para medir a habilidade de adolescentes e adultos em organizar estímulos visuais e, desta forma, explorar a existência de qualquer dificuldade na discriminação visual. No entanto, esta pesquisa envolveu apenas adultos jovens de 18 anos, o que limita sua aplicabilidade para indivíduos mais velhos (Toselo, 2005).

As dimensões dos objetos, a noção de profundidade, as relações figura-fundo são aspectos que interagem entre si e se complementam para proporcionar o equilíbrio necessário na identificação de um objeto. De modo que, havendo déficit ou alteração em algum destes atributos, os demais também irão se mostrar alterados. Ou seja, alterações visuais na percepção de objeto ou na coordenação visuo-motora acarretarão distúrbios de atenção, estabilidade e adaptação ao meio (Goldstein, 2005).

Apesar da importância de se estudar a percepção visual de indivíduos adultos, a maioria dos instrumentos elaborados é voltada para avaliação de crianças. Além disso, os estudos realizados no Brasil utilizam instrumentos cujas propriedades psicométricas são desconhecidas, tendo sido, muitas vezes, desenvolvidos em estudos internacionais, o que dificulta seu emprego para população brasileira. Baseado nestes preceitos, este trabalho teve como objetivo construir e conhecer os parâmetros psicométricos de uma medida de avaliação da percepção visual para adultos, a Escala de Avaliação da Percepção Visual (EAPV).

A Escala de Avaliação da Percepção Visual (EAPV) foi baseada no instrumento desenvolvido por Dias e Chaves (2000a), intitulado Programa de Treinamento da Percepção Visual (PTPV). O PTPV foi escolhido dentre os vários instrumentos referenciados na literatura, por (a) tratar-se de um teste originalmente elaborado na língua portuguesa, o que facilita o processo de adaptação cultural e semântica; (b) corresponder a um programa baseado em estudos já realizados sobre a implementação de programas envolvendo esta temática em vários países (Dias, 2007) e (c) ser fundamentado nos princípios postulados por Frostig (1964) e Luria (1987), envolvendo aspectos de avaliação da percepção visual, como constância da forma, fechamento visual, percepção figura-fundo e orientação espacial.

Estes predicados são os princípios norteadores da Teoria da Gestalt, uma das principais teorias da percepção da forma. Seus alicerces estão na lei da pregnância da forma, isto é, todas as formas tendem a ser percebidas em seu caráter mais simples. Segundo a Gestalt, o importante é perceber a forma por ela mesma, vê-la como "todo" estruturado, resultantes de relações entre as partes do todo (Gomes, 2000). Outros testes também incluem os parâmetros referidos anteriormente como é o caso do DTVP clássico e suas modalidades (DTVP-2 e DTVP-A), amplamente empregados em avaliações clínicas e pesquisas científicas envolvendo análise da percepção visual (Gray, 2006; Ito et al., 2008; Ziviani, Copley, Ownsworth, Campbell, \& Cummins, 2008).

O embasamento lógico subjacente à escolha das categorias componentes da EAPV é também fundamentado nos estudos de diversos investigadores dessa temática (Chalfant \& Scheffelin, 1969; Deliberato \& Gonçalves, 2003; Gabbard, 1992), que consideram várias categorias da percepção visual inseridas na Teoria da Gestalt e em consonância ao defendido por Frostig (1964). Esta consistência teórica foi a base da decisão dos autores para designar os subtestes da EAPV. 
Dessa forma, o presente trabalho compreende dois estudos, orientados pelos seguintes objetivos: (a) Construção da Escala de Avaliação da Percepção Visual (EAPV), (b) Análise das Propriedades Psicométricas da EAPV.

\section{Estudo 1 - Construção da Escala de Avaliação da Percepção Visual}

\section{Método}

Amostra. A amostra foi do tipo não probabilística, composta por 295 acompanhantes de usuários de serviços de saúde, com idades entre 35 e 65 anos $(M=46,21$ e $D P=3,40)$, declarando-se pertencer à classe sócio-econômica média $(53,4 \%)$ ou média baixa $(46,6 \%)$. Os participantes apresentaram grau de escolaridade variando entre o ensino fundamental $(54,2 \%)$, médio $(41,8 \%)$ e superior $(5,8 \%)$.

Instrumento. A EAPV foi baseada no instrumento desenvolvido por Dias e Chaves (2000a), intitulado Programa de Treinamento da Percepção Visual (PTPV), aplicado em crianças com dificuldade de aprendizagem.

$\mathrm{O}$ instrumento original possui 64 itens que avaliam oito categorias da percepção visual: coordenação óculo manual, cópia, relações espaciais, posição no espaço, figura-fundo, velocidade visual motora, lacunas visuais e constância da forma. Foi desenvolvido, originalmente, para o treino dessas competências da percepção visual em crianças com deficiência de aprendizagem.

Para a operacionalização do construto, foram seguidos critérios estabelecidos por Pasquali (2001), como simplicidade, clareza, relevância, precisão, variedade, tipicidade, modalidade, credibilidade, amplitude e equilíbrio. Dessa forma, foi elaborada uma versão preliminar da escala, com 40 itens, a partir do instrumento original, PTPV, sendo suas respostas pontuadas como correto (1), errado (0) ou faltante (0).

Procedimentos. Após o contato e consentimento dos participantes, foi efetuada a aplicação do instrumento. Esta atividade contou com a colaboração de três examinadores previamente instruídos para intervirem o mínimo possível. Os questionários foram aplicados individualmente, em ambiente arejado, bem iluminado e livre de ruídos. A aplicação do instrumento foi realizada em um tempo médio de 25 a 35 minutos. Todos os voluntários assinaram Termo de Consentimento Livre e Esclarecido no qual eram informados sobre o protocolo da pesquisa e o objeto do trabalho.

\section{Resultados}

Seleção dos Itens. A partir da análise fatorial com extração de fatores pelo critério de Kaiser (1958), que propõe considerar apenas os autovalores superiores a um, foram obtidos alguns fatores saturados em cinco itens.
Conforme sugerem estudos da literatura (Ford, MacCallum, \& Tait, 1986; Rego, 2000), foram retirados estes fatores, assim como aqueles itens cujas saturações foram superiores a $0,40 \mathrm{em}$ mais de um fator e aqueles cuja diferença entre as duas saturações mais elevadas era inferior a 0,20 . Este procedimento permitiu a formação de uma escala tetrafatorial constituída por 20 itens que avaliam quatro dimensões referentes à percepção visual. Nos 4 subtestes, cada um dos itens foi pontuado como correto (1), errado (0) ou faltante (0). Assim, em cada subteste o escore mínimo é zero e o máximo corresponde ao total de itens do subteste. Desse modo, a versão final da EAPV, cuja pontuação total varia de 0 a 20 pontos, foi constituída pelas seguintes categorias:

Constância da forma (itens 1, 5, 9, 13 e 17): envolve o reconhecimento de características dominantes de certas figuras ou formas, quando aparecem em diferentes sombreados, texturas, tamanhos e posições. Para cada resposta correta, é atribuído 1 ponto, assim sua pontuação varia de 0 a 5 pontos. A Figura 1 mostra um dos itens deste teste.
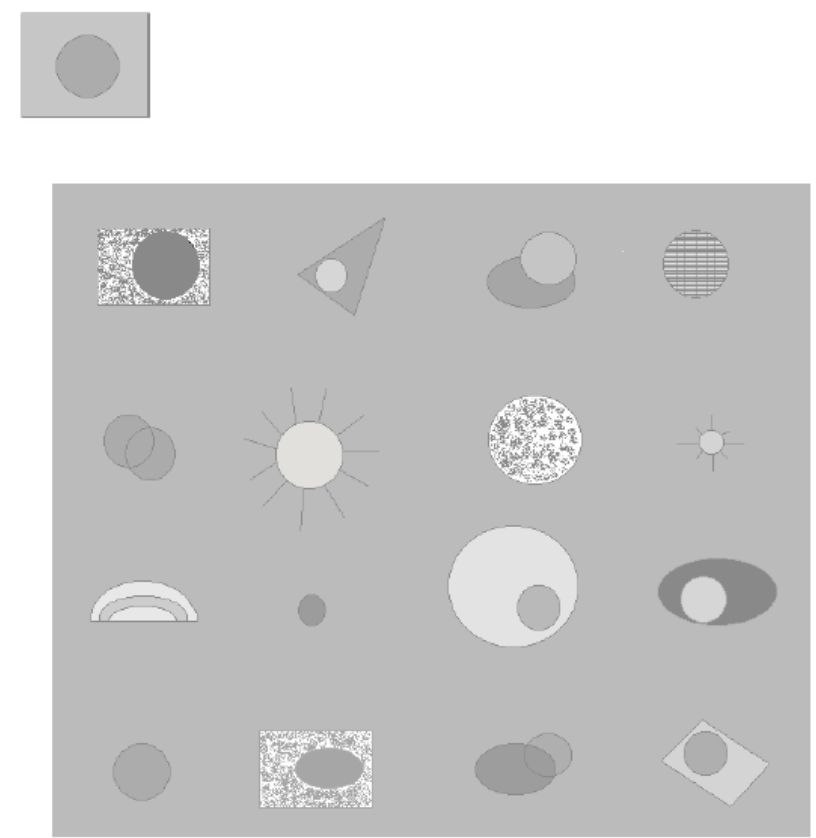

Figura 1. Exemplo de Item que Compõe o Teste Constância da Forma.

Figura-fundo (itens 2, 6, 10, 14 e 18): envolve o reconhecimento de figuras inseridas em um fundo sensorial comum. É atribuído 1 ponto para cada acerto. Sua pontuação varia de $0-5$ pontos. A Figura 2 mostra um exemplo de item deste teste.

Posição no espaço (itens 3, 7, 11, 15 e 19): envolve a discriminação das reversões e rotações de figuras. Para cada resposta correta, é atribuído 1 ponto, desse modo sua pontuação varia de 0 a 5 pontos;

Relações espaciais (itens 4, 8, 12, 16 e 20): envolve a análise de formas e padrões em relação a um corpo e espa- 


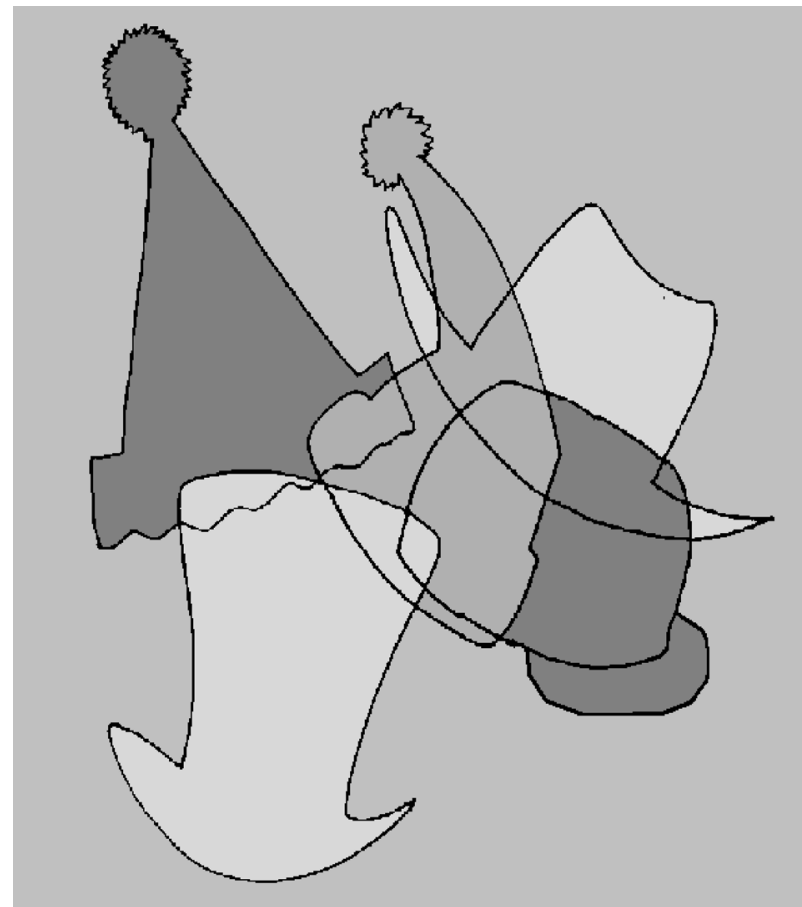

Figura 2. Exemplo de Item que Compõe o Teste Figura-Fundo.

ço. É atribuído 1 ponto para cada acerto, podendo sua pontuação variar de $0-5$ pontos.

\section{Análise de Juizes}

Para a condução da análise de juízes, foi elaborado um formulário contendo a definição operacional do constructo, os itens propostos e as instruções para sua análise, baseado no protocolo desenvolvido por Cerqueira e Nascimento (2008). A avaliação dos itens incluiu os seguintes quesitos: (a) adequação do conteúdo, (b) identificação do lócus do controle, (c) a pertinência, (d) a relevância, e (e) a adequação da formulação. $\mathrm{O}$ formulário também incluiu espaço para que os juízes apresentassem sugestões.

Para a análise de juízes, foi estruturada uma equipe formada por profissionais de saúde com conhecimento da língua portuguesa e larga experiência na aplicação de escalas de percepção e atendimento a pacientes com distúrbios cognitivos e visuais. Estes profissionais receberam o formulário de avaliação dos itens propostos e os dados foram analisados considerando cada um dos quesitos avaliados e as sugestões de reformulação dos componentes. O critério para a manutenção do item na escala foi a obtenção de pelo menos $80 \%$ de concordância entre os juízes em cada um dos quesitos (Pasquali, 2001). Houve consenso quanto à adequação da escala para população brasileira, sendo todos os itens mantidos.

\section{Análise Semântica}

Após a análise dos juízes, procedeu-se à análise semântica conforme protocolo desenvolvido por Paschoal e Tamayo (2008). O objetivo desta etapa foi verificar a compreensão dos itens por membros da população-alvo. $\mathrm{O}$ instrumento foi aplicado em seis voluntários divididos em dois grupos de três indivíduos. Todos os participantes eram acompanhantes de usuários de serviços públicos de saúde, apresentavam boa saúde física e acuidade visual normal ou corrigida. Os voluntários possuíam nível sócio-econômico médio e grau de escolaridade variando entre ensino fundamental $(N=2)$, médio $(N=3)$ e superior $(N=1)$. A escala foi aplicada individualmente e após o término foi solicitado aos voluntários que apontassem suas dificuldades em relação às instruções e aos termos presentes nos itens. As instruções foram compreendidas por todos os participantes tais como apresentadas e foram mantidas. Nenhum item foi modificado.

\section{Estudo 2- Análise das Propriedades Psicométricas da EAPV}

\section{Método}

Participantes. A amostra foi constituída por 287 voluntários, acompanhantes de usuários de serviços de saúde da rede pública, convidados a participar da pesquisa enquanto aguardavam o atendimento de seus familiares em Postos Municipais de Saúde. A seleção da amostra foi não probabilística, do tipo intencional.

Foi aplicado o Mini Exame de Estado Mental (MEEM) para assegurar a inclusão de indivíduos saudáveis no estudo. O MEEM é composto por cinco dimensões (concentração, linguagem/práxis, orientação, memória e atenção), amplamente utilizado para detecção e acompanhamento da evolução de alterações cognitivas (Valle, Castro-Costa, Firmo, Uchoa, \& Lima-Costa, 2009). Os voluntários também foram avaliados através da Cumullative Ilness Research Scale (CIRS), que investiga a presença de 14 "conjuntos" de doenças (cardíaca; vascular; hematológica; respiratória; oftalmológica; gastrointestinal alta e gastrointestinal baixa; hepática e pancreática; renal; geniturinária, musculoesquelética e tegumentar; neurológica; endócrina e metabólica; mamária; e psiquiátrica). A CIRS considera as situações de ausência, leve, moderada, severa ou extremamente severa para cada "conjunto", com pontuações que variam de 0-4 (Navega \& Oishi, 2007). A acuidade visual foi medida com a cartela de optotipos "E" de Rasquin.

A perda amostral $(36,23 \%)$ foi maior do que a prevista devido ao número de participantes pertencentes a um ou mais critérios de exclusão desta pesquisa: (a) acuidade visual prejudicada ou não-corrigida, (b) distúrbios cognitivos, (c) doenças associadas, (d) disfunções limitantes para a realização das provas, (e) distúrbios psiquiátricos, (f) doenças oculares e (g) uso de medicamentos que modulam a atividade do sistema nervoso central.

Desse modo, este trabalho analisou 183 indivíduos saudáveis sendo 106 mulheres e 77 homens, com faixa etária entre 35-65 anos $(M=47,98$ e $D P=7,47)$ e nível 
sócio-econômico médio $(69,9 \%)$ e médio-baixo $(30,1 \%)$. $\mathrm{O}$ grau de escolaridade dos participantes distribuiu-se entre o ensino fundamental $(54,1 \%)$, médio $(43,7 \%)$ e superior $(2,2 \%)$ [Tabela 1$]$.

Tabela 1

Dados Sócio-demográficos dos Participantes

\begin{tabular}{lrr}
\hline Categorias & Mulheres $F(\%)$ & Homens $F(\%)$ \\
\hline Faixa Etária & & \\
35-45 anos & $41(38,67 \%)$ & $32(41,55 \%)$ \\
46-55 anos & $43(40,56 \%)$ & $25(32,46 \%)$ \\
56-65 anos & $22(20,75 \%)$ & $20(25,97 \%)$ \\
Escolaridade & & \\
Primeiro Grau & $66(36,06 \%)$ & $33(42,85 \%)$ \\
Segundo Grau & $38(20,76 \%)$ & $42(54,54 \%)$ \\
Terceiro Grau & $2(1,09 \%)$ & $2(2,59 \%)$ \\
\hline Total & $106(57,92 \%)$ & $77(42,07 \%)$ \\
\hline
\end{tabular}

\section{Procedimentos de Coleta de Dados}

O presente estudo é uma pesquisa descritiva, com corte transversal, realizada em Unidades de Saúde e Centros de Tratamento Municipais, no período de outubro a dezembro de 2009. Para sua execução, o estudo seguiu as recomendações do Conselho Nacional de Saúde (Resolução 196/96 e suas complementares) e foi submetido à avaliação e aprovação do Comitê de Ética em Pesquisa da Instituição. Os participantes assinaram um Termo de Consentimento Livre e Esclarecido autorizando a realização e a publicação do estudo.

Após as avaliações iniciais, realizadas com o MEEM e a CIRS, os participantes foram convidados a preencher um questionário com perguntas relativas a dados sóciodemográficos. Em seguida, foi realizada a avaliação da percepção visual utilizando a EAPV.

Os dados foram coletados individualmente, em local silencioso, bem iluminado, arejado e livre de interrupções exteriores. As instruções e as questões da escala eram lidas pelo examinador. Os voluntários foram orientados sobre o preenchimento do instrumento e estimulados a informar ao examinador sobre eventuais dúvidas. Ao final da aplicação foi fornecido endereço e telefone através dos quais os participantes poderiam obter informações sobre os resultados do estudo. A coleta de dados teve duração média de 15 a 25 minutos.

\section{Estudo Psicométrico}

Os dados obtidos foram submetidos à análise estatística, utilizando o pacote estatístico SPSS (Statistical Package for the Social Sciences), versão 16.0 para Windows. Para o estudo psicométrico foi realizada avaliação da confiabilidade e validade fatorial do instrumento.

A avaliação da confiabilidade foi medida através do Alfa de Cronbach para comprovação da consistência interna e de aplicação do teste-reteste para análise da estabilidade temporal da escala. O valor mínimo de 0,70 foi recomendado por Rowland, Arkkelin e Crisler (1991) para considerar que os itens avaliam consistentemente o mesmo constructo. Valores de alfa altos, no entanto, são necessários, mas não suficientes, uma vez que é uma estimativa "otimista" da confiabilidade (Streiner \& Norman, 1995). A fidedignidade por meio do teste-reteste foi realizada com 171 indivíduos $(94,44 \%$ do total de participantes), sendo o intervalo de tempo entre as duas aplicações não superior a 14 dias. O Coeficiente de Correlação Intra-Classe (ICC) foi utilizado para verificar a concordância teste-reteste de escores da escala.

Para o tratamento dos dados, as respostas faltantes foram repontuadas como zero. Na investigação de evidências de validade, foram realizadas análises fatoriais exploratórias com o objetivo de avaliar o grau de representatividade dos constructos. Inicialmente, foi verificada a fatorabilidade da matriz gerada. Foram calculados os valores do teste de esfericidade de Bartlett (AIC) e do Kaiser-Meyer-Olkin (KMO). E para a rotação e extração dos fatores foi utilizado o método PAF (Principal Axis Factoring) com rotação oblíqua. O pressuposto inicial foi de que o instrumento seria formado por quatro fatores. A fim de assegurar que cada item representava o constructo subjacente ao fator, foi estipulada uma carga fatorial mínima de 0,40 para aceitar o item.

\section{Resultados}

$\mathrm{Na}$ análise da consistência interna, o coeficiente Alfa de Cronbach variou de 0,84 a 0,93 nas 4 sub-escalas, apontando que o instrumento apresenta adequada consistência interna (Tabela 2).

Quanto à reprodutibilidade, não houve diferença estatisticamente significativa nas médias dos escores, quando foram considerados o momento 1 (teste) e o momento 2 (reteste). Os coeficientes de correlação entre os escores dos momentos 1 e 2 foram significativos, variando de $0,88(p<0,001)$ a $0,95(p<0,001)$.

Em relação à análise fatorial, $\mathrm{O} \mathrm{KMO}$ encontrado foi de 0,89 e o teste de esfericidade de Bartlett indicou valor de 5195,50 $(p<0,001)$, ambos apontando para a fatorabilidade da matriz. Obteve-se uma estrutura tetrafatorial, que no seu conjunto explica $57,52 \%$ da variância (Tabela 2).

$\mathrm{O}$ instrumento ficou composto por quatro fatores. $\mathrm{O}$ primeiro, constância da forma, com cinco itens e Alfa de Cronbach de 0,93, explica $25,78 \%$ da variância; o segundo fator, figura-fundo, com cinco itens e Alfa de Cronbach de 0,89 , explica $14,65 \%$ da variância; o terceiro fator, posição no espaço, com cinco itens e Alfa de Cronbach de 0,86 , explica $9,62 \%$ da variância; por fim, o fator relação espacial, com cinco itens e Alfa de Cronbach de 0,84, explica 7,47\% da variância. Juntos, os quatro fatores explicam $57,52 \%$ da variância do constructo. 
Andrade, S. M., Dias, M. M. C. B. S., Oliveira, E. A., Locks Neto, F., Nogueira, R. M. T. B. L. \& Santos, N. A. (2012). Construção e Evidências Psicométricas de uma Escala de Avaliação da Percepção Visual.

Tabela 2

Matriz Fatorial dos Testes e Precisão dos Fatores

Item

Fator 1 Fator 2 Fator 3 Fator 4

1. Assinale as formas que têm o mesmo formato do retângulo acima

0,898
0,864
0,777
0,763
0,648

5. Indique que figuras são iguais à destacada

9. Marque as figuras que têm formato idêntico

13. Aponte quais as formas são iguais àquela destacada acima

2. Indique, na figura, que animais estão representados

0,819

0,781

6. Aponte, na figura, os chapéus representados em sobreposição

0,759

10. Assinale, na figura, as ovelhas representadas em sobreposição

0,686

14. Marque na figura as formas que você vê no retângulo de cima

0,632

3. Aponte qual a seta está na mesma posição daquela situada no primeiro quadro

7. Marque qual a figura está na mesma posição dos símbolos à esquerda

11. Assinale, em cada fileira, os desenhos que estão na mesma posição

15. Indique quais os quadrados apresentam figuras na mesma posição

19. Mostre qual a figura está na mesma posição dos retângulos à esquerda

4. Marque, nos retângulos com pontos, traços na mesma posição dos do retângulo inicial

8. Una os pontos como demarcado no primeiro quadro

12. Ligue os pontos formando três figuras iguais à primeira

16. Faça, em cada quadro, traços formando uma figura igual à primeira

20. Junte os pontos para que fiquem igual ao primeiro desenho

Eigenvalue

\% da Variância

Total de itens

Alfa de Cronbach

\begin{tabular}{rrrr}
7,258 & 3,431 & 2,441 & 1,769 \\
25,783 & 14,658 & 9,623 & 7,471 \\
5 & 5 & 5 & 5 \\
0,933 & 0,892 & 0,867 & 0,840 \\
\hline
\end{tabular}

$\mathrm{O}$ instrumento ficou composto por quatro fatores. $\mathrm{O}$ primeiro, constância da forma, com cinco itens e Alfa de Cronbach de 0,93 , explica $25,78 \%$ da variância; o segundo fator, figura-fundo, com cinco itens e Alfa de Cronbach de 0,89 , explica $14,65 \%$ da variância; o terceiro fator, posição no espaço, com cinco itens e Alfa de Cronbach de 0,86 , explica $9,62 \%$ da variância; por fim, o fator relação espacial, com cinco itens e Alfa de Cronbach de 0,84, explica 7,47\% da variância. Juntos, os quatro fatores explicam $57,52 \%$ da variância do constructo.

\section{Discussão e Considerações Finais}

De acordo com Pasquali (2001), os testes psicológicos são considerados instrumentos de medida e, por isso, devem apresentar algumas características para serem confiáveis. As características consideradas mais importantes dizem respeito à validade e à precisão do instrumento.

O Programa de Treino da Percepção Visual (PTPV), no qual foi baseada a EAPV, foi originalmente elaborado para aplicação em crianças, na faixa etária dos 6 a 9 anos, com dificuldades de aprendizagem. As aferições empregadas no estudo original relacionadas à equivalência entre os itens, equivalência operacional e validade de conteúdo, realizadas por meio de avaliação de juízes, apresentaram consonância com os dados obtidos no presente trabalho. Segundo Dias (2007), os resultados demonstraram que os itens do PTPV são fáceis de serem compreendidos; estão redigidos com clareza; avaliam aspectos importantes e têm potencial para discriminar componentes alterados da percepção visual. Conquanto este instrumento tenha sido aplicado previamente em uma amostra de 353 alunos de escolas do Conselho de Braga, em Portugal, nenhuma informação foi encontrada sobre sua validade de construto e consistência interna.

A Escala de Avaliação da Percepção Visual apresentou parâmetros psicométricos satisfatórios, boa consistência interna, concordância interjuízes, adequação semântica, significância no teste-reteste e evidências de validade de constructo. Em síntese, os cálculos permitiram confirmar a adequação da validade fatorial e consistência interna da medida. Os resultados sugerem a coerência do modelo tetrafatorial proposto.

Teixeira (2008), em um trabalho envolvendo análise da percepção de formas e espaço em 35 participantes com distúrbios neurológicos, verificou que todos os pacientes quando comparados ao grupo controle apresentaram 
dificuldade na discriminação da forma de objetos. Estes resultados são semelhantes aos encontrados por Parmentes, Weinstock-Guttman, Gang, Munschauer e Benedict (2007), em pacientes com Esclerose Múltipla, e por Haberecht et al. (2001), em indivíduos com Síndrome de Turner, o que evidencia a importância de analisar a dimensão constância da forma, podendo ser este um marcador clínico do quadro evolutivo de pacientes com deficiências na identificação de objetos.

Em um estudo semelhante ao da presente pesquisa, Gomes e Borges (2009) avaliaram as propriedades psicométricas do Conjunto de Testes de Habilidade VisuoEspacial, em adultos jovens, e verificaram a associação de aspectos visuo-motores intimamente ligados a parâmetros como memória e fechamento visual, sendo este último um aspecto importante na avaliação e caracterização do perfil perceptivo-visual do indivíduo.

Em relação à discriminação figura-fundo, posição e relação espacial, os estudos de Ribeiro (2006) demonstram que a organização espacial, envolvendo parâmetros como posição e relações espaciais do objeto, encontra-se relacionada a outros aspectos da percepção, a exemplo da discriminação figura-fundo. Em seu trabalho, o autor sugere a existência de possíveis alterações na percepção visual de luminosidade a partir de variações físicas dos estímulos, com base na organização espacial de figurafundo criada pela associação dos efeitos ilusórios de contraste e contornos subjetivos.

O presente trabalho apresenta algumas limitações, como a forma de seleção da amostra. Sugere-se, portanto, o fomento de pesquisas na área utilizando amostragem probabilística. Aponta-se também a necessidade de futuras investigações para orientar construções de versões da EAPV voltadas para áreas da percepção como lateralidade e esquema corporal, presentes em estudos multifatoriais, que se relacionam com o desempenho do indivíduo na discriminação dos objetos e que podem interferir na análise dos parâmetros perceptivos visuais ou motores (Freitas, 2008). Além disso, apesar dos resultados favoráveis da análise fatorial, esta é uma técnica de natureza exploratória, sendo, deste modo, necessários novos empreendimentos envolvendo amostras clínicas e o emprego de outras técnicas de análises estatísticas, como a análise fatorial confirmatória, através dos modelos de equação estrutural (Hair, Black, Babin, Anderson, \& Tatham, 2009).

A partir dos dados obtidos, verifica-se que este estudo apresenta contribuições substantivas. A EAPV pode ser considerada um instrumento eficaz na avaliação das dimensões componentes da percepção visual, em diversas áreas de aplicação do campo das ciências psicológicas, psicométricas, neurológicas e cognitivas. Além disso, demonstrou ser um teste de simples aplicação e levantamento, facilitando a aplicabilidade na prática clínica. Diante dos custos para realizar outros tipos de exames que avaliam tais funções, a Escala de Avaliação da Percepção Visual mostra-se eficaz, fidedigna e de rápida aplicação.

\section{Referências}

Bandeira, R. D., \& Hutz, C. S. (1994). A contribuição dos testes DFH, Bender e Raven na predição do rendimento escolar na primeira série. Psicologia: Teoria e Pesquisa, 10(1), 59-72.

Bartholomeu, D., Rueda, F. J. M., \& Sisto, F. F. (2005). Teste de Bender e dificuldades de aprendizagem: Quão válido é o sistema Koppitz? Avaliação Psicológica, 4, 13-21.

Caixeta, L., \& Nitrini, R. (2001). Clinical subtypes of frontotemporal dementia. Arquivos de Neuro-Psiquiatria, 59(3A), 577-581.

Cerqueira, M., \& Nascimento, E. (2008). Construção e validação da Escala de Lócus de Controle Parental na Saúde. PsicoUSF, 13(2), 253-263.

Chalfant, J., \& Scheffelin, M. (1969). Central processing dysfunctions in children: A review of research. Bethesda, MD: Department of Health, Education and Welfare.

Deliberato, D. (2000). Aspectos da percepção visual em préescolares surdos e ouvintes. (Tese de Doutorado não-publicada). Universidade Estadual de Campinas, SP.

Deliberato, D., \& Gonçalves, V. (2003). Aspectos do desenvolvimento da percepção visual. Temas sobre Desenvolvimento, 12(67), 5-10.

Dias, M. (2007). A utilização da imagem e das tecnologias interactivas nos programas de treino da percepção visual: Um estudo com alunos do $1^{\circ}$ ciclo do ensino básico com dificuldades de aprendizagem. (Tese de Doutorado nãopublicada). Universidade do Minho, Braga, Portugal.

Dias, M. (2009). A utilização das tecnologias interactivas nos programas de treino da percepção visual: Um estudo com alunos do $1{ }^{\circ}$ ciclo do ensino básico com dificuldades de aprendizagem. In Anais do X Congresso Internacional Galego-Português de Psicopedagogia (pp. 6033-6046). Braga, Portugal: Universidade do Minho.

Dias, M., \& Chaves, J. (2000a). Percepção visual e dificuldades de aprendizagem: Um estudo com alunos do $1^{\circ}$ ciclo do Ensino Básico. Revista Galego-Portuguesa de Psicoloxía e Educacion, 6, 389-398.

Dias, M., \& Chaves, J. (2000b). Percepção visual e dificuldades de aprendizagem: Um estudo com alunos do $1^{\circ}$ Ciclo do Ensino Básico. In Libro de Actas do V Congreso GalegoPortuguês de Psicopedagoxía (pp. 389-398). Santiago de Compostela, Espanha.

Dias, M., \& Chaves, J. (2001). O Programa de Treino da Percepção Visual para alunos com dificuldades de aprendizagem do $1^{\circ}$ ciclo do Ensino Básico. In Resumos da II Conferência Internacional de Tecnologias de Informação e Comunicação na Educação (pp. 707-721). Braga, Portugal: Universidade do Minho.

Ford, J., MacCallum, R., \& Tait, M. (1986). The application of exploratory factor analysis in applied psychology: A critical review and analysis. Personnel Psychology, 39, 292-314.

Forgus, R. (1971). Percepção: O processo básico do desenvolvimento cognitivo. São Paulo, SP: Editora da Universidade de São Paulo.

Freitas, N. (2008). Esquema corporal, imagem visual e representação do próprio corpo. Ciências \& Cognição, 13(3), 318324.

Frostig, M. (1964). The Marianne Frostig Developmental Test of Visual Perception. Palo Alto, CA: Consulting Psychologist Press.

Frostig, M., \& Muller, H. (1986). Discapacidades especificas de aprendizaje em ninos: detección y tratamiento. Buenos Aires, Argentina: Médica Panamericana. 
Andrade, S. M., Dias, M. M. C. B. S., Oliveira, E. A., Locks Neto, F., Nogueira, R. M. T. B. L. \& Santos, N. A. (2012). Construção e Evidências Psicométricas de uma Escala de Avaliação da Percepção Visual.

Gabbard, C. (1992). Lifelong motor development. Dubuque, IA: Wm C. Brown.

Goldstein, B. (2005). Blackwell handbook of sensation and perception. Oxford, UK: Blackwell.

Gomes, C., \& Borges, O. (2009). Propriedades psicométricas do conjunto de testes da habilidade visuo espacial. PsicoUSF, 14(1), 19-34.

Gomes, J. (2000). Gestalt do objeto: Sistema de leitura visual da forma. São Paulo, SP: Escrituras.

Granzoto, J., Ostermann, C., Brum, L., Pereira, P., \& Granzoto, T. (2003). Avaliação da acuidade visual em escolares da $1^{\text {a }}$ série do ensino fundamental. Arquivos Brasileiros de Oftalmologia, 66(2), 167-171.

Gray, R. (2006). Comprehensive Trail Making Test. Journal of Psychoeducational Assessment, 24(1), 88-91.

Haberecht, M., Menon, V., Warsofsky, I., White, C., DyerFriedman, J., Glover, G., et al. (2001). Functional neuroanatomy of visual-spatial working memory in Turner syndrome. Human Brain Mapping, 14, 96-107.

Hair, J., Black, W., Babin, B., Anderson, R., \& Tatham (2009). Análise multivariada de dados. Porto Alegre, RS: Bookman.

Hammill, D., Pearson, N., \& Voress, J. (1993). Development Test of Visual Perception. Austin, TX: Pro-Ed.

Ito, J., Saijo, H., Araki, A., Tanaka, H., Tasaki, T., Cho, K., et al. (2008). Assessment of visuoperceptual disturbance in children with spastic diplegia using measurements of the lateral ventricles on cerebral MRI. Developmental Medicine \& Child Neurology, 38(6), 496-502.

Kaiser, H. (1958). The Varimax Criterion for Analytic Rotation in Factor Analysis. Psychometrika, 23(3), 187-200.

Kaiser, M., Albaret, J., \& Doudin, P. (2009). Relationship between visual-motor integration, eye-hand coordination, and quality of handwriting. Journal of Occupational Therapy, Schools \& Early Intervention, 2(2), 87-95.

Luria, A. (1987). Pensamento e linguagem: As últimas conferências. Porto Alegre, RS: Artes Médicas.

Moryosef-Ittah, S., \& Hinojosa, J. (2006). Discriminant validity of the Developmental Test of Visual Perception-2 for children with learning disabilities. Occupational Therapy International, 3(3), 204-211.

Navega, M., \& Oishi, J. (2007). Comparação da qualidade de vida relacionada à saúde entre mulheres na pós-menopausa praticantes de atividade física com e sem osteoporose. Revista Brasileira de Reumatologia, 47(4), 258-264.

Noronha, A., \& Primi, R. (2005). Instrumentos de avaliação mais conhecidos/utilizados por psicólogos e estudantes de psicologia. Psicologia: Reflexão e Crítica, 18(3), 390-401.

Oliveira, C., Rodrigues, J., \& Fonseca, R. (2009). O uso de instrumentos neuropsicológicos na avaliação de dificuldades de aprendizagem. Revista de Psicopedagogia, 26(79), 65-76.

Oliveira, F., \& Rodrigues, S. (2005). Críticas Gibsonianas à perspectiva representacionista da percepção visual. Ciências \& Cognição, 6(1), 98-108.

Oliveira, M., Rigoni, M., Andretta, I., \& Moraes, J. (2004). Validação do Teste Figuras Complexas de Rey na população brasileira. Avaliação Psicológica, 3(1), 33-38.

Parmentes, B., Weinstock-Guttman, B., Gang, N., Munschauer, F., \& Benedict, R. (2007). Screening for cognitive impairment in multiple sclerosis using the symbol digit modalities. Multiple Sclerosis, 13, 52-57.

Paschoal, T., \& Tamayo, A. (2008). Construção e validação da Escala de Bem-estar no Trabalho. Avaliação Psicológica, 7(1), 11-22.

Pasquali, L. (2001). Técnicas de exame psicológico - TEP: Manual. São Paulo, SP: Casa do Psicólogo.
Pinelli, B., Jr., \& Pasquali, L. (1990). Parâmetros psicométricos do Teste Gestaltico Visomotor de Bender: Um estudo empírico. Revista de Psicologia, 1/2(7/8), 51-74.

Posada, M. C. (2002). Un sistema alternativo para la evaluación del Test Gestáltico Viso-motor de Bender. Revista Ibero-americana de Diagnóstico y Evaluación Psicológica, 14(2), 9-31.

Quental, N., Brucki, S., \& Bueno, O. (2009). Visuospatial function in early Alzheimer's disease: Preliminary study. Dementia \& Neuropsychologia, 3(3), 234-240.

Rego, A. (2000). Os motivos de sucesso, afiliação e poder: Desenvolvimento de um instrumento de medida. Análise Psicológica, 3(XVIII), 335-344.

Reynolds, C., Pearson, N., \& Voress, J. (2002). Developmental Test of Visual Perception-Adolescent and Adult (DTVP-A). Austin, TX: Pro-Ed.

Ribeiro, A. (2006). Organização espacial na percepção visual de luminosidade. (Tese de Doutorado não-publicada). Instituto de Psicologia, Universidade de Brasília, DF.

Rowland, D., Arkkelin, D., \& Crisler, L. (1991). Computerbased data analysis: Using SPSS in the Social and Behavioral Sciences. Chicago, IL: Wardsworth.

Santos, A., \& Jorge, L. (2007). Teste de Bender com disléxicos: Comparação de dois sistemas de pontuação. PsicoUSF, 12(1), 13-21.

Silva, R., \& Nunes, M. (2007). Teste Gestáltico Visomotor de Bender: Revendo sua história. Avaliação Psicológica, 6(1), 77-88.

Sisto, F. F., Bueno, J. M. H., \& Rueda, F. J. M. (2003). Traços de personalidade na infância e distorção e integração de formas: Um estudo de validade. Psicologia em Estudo, 8(1), 77-84.

Sisto, F. F., Noronha, A. P. P., \& Santos, A. A. A. (2006). Teste Gestaltico Visomotor de Bender - Sistema de Pontuação Gradual (B-SPG). São Paulo, SP: Vetor.

Streiner, D., \& Norman, G. (1995). Health measurement scales: A practical guide to their development and use. London: Oxford University Press.

Teixeira, R. (2008). Estudo da visão de cores, percepção de formas e espaço em pacientes com esclerose múltipla. (Dissertação de Mestrado não-publicada). Instituto de Psicologia, Universidade de São Paulo, SP.

Toni, P. (2010). Parâmetros psicométricos dos Cubos de Kohs na avaliação neuropsicológica das funções executivas. (Tese de Doutorado não-publicada). Instituto de Psicologia, Universidade de São Paulo, SP.

Toselo, D. (2005). Contribuição para o estudo normativo do Hooper Visual Organization Test (VOT). Psicologia Hospitalar, 3(1), 59-83.

Valle, E., Castro-Costa, E., Firmo, J., Uchoa, E., \& Lima-Costa, M. (2009). Estudo de base populacional dos fatores associados ao desempenho no Mini Exame do Estado Mental entre idosos: Projeto Bambuí. Cadernos de Saúde Pública, 25(4), 918-926.

Ziviani, J., Copley, J., Ownsworth, T., Campbell, N., \& Cummins, K. (2008). Visual perception abilities and executive functions in children with school-related occupational performance difficulties. Journal of Occupational Therapy, Schools \& Early Intervention, 1(3-4), 246-262.

Recebido: 29/04/2010

$1^{a}$ revisão: $04 / 11 / 2010$ Aceite final: $11 / 11 / 2010$ 